\title{
A polymorphism against prions
}

Transmissible spongiform encephalopathies (TSEs) can go by many names. Sheep and goats get scrapie. In cervids (deer, moose, and elk), it's called chronic wasting disease. Cattle "go mad" with bovine spongiform encephalopathy. Even humans have variants; the most common, Creutzfeldt-Jakob disease, afflicts $1-5$ in a million people each year.

The underlying cause of the different manifestations of these rare but fatal neurodegenerative diseases is a thought to be a single agent: prion proteins. Their purpose in health is still under investigation, but prions have an unfortunate proclivity to misfold and an apparent resistance to the proteases that would normally degrade abnormal proteins. The initial spark is still unclear, but when one prion misfolds, it causes other nearby prion proteins to do so as well. This chain reaction propagates through an animals' brain, causing TSEs' characteristic sponge-like holes and progressively destroying normal brain function. Infectious prions can spread from animal to animal, and also from species to species.

But not all animals are equally susceptible to prion diseases. Understanding transmission barriers between species is an important area of research for scientists like Joaquín Castilla, a research professor at CIC bioGUNE in Spain. Castilla explains that prions come in different three dimensional structures, or strains; some can cross species barriers and cause infections while others cannot. For example, humans can become sick from consuming sick cows, but not sick deer (at least so far). Castilla and his lab want to understand why, in the hopes that identifying mammals that are resistant to infection by different prion strains could lead to potential treatments. Currently, TSEs are untreatable.

Over the years, Castilla has been looking closely at the normal prion proteins of animals thought to be resistant. Two candidates, rabbits and horses, have been scratched off the list. But a thirdcanines-still holds potential.

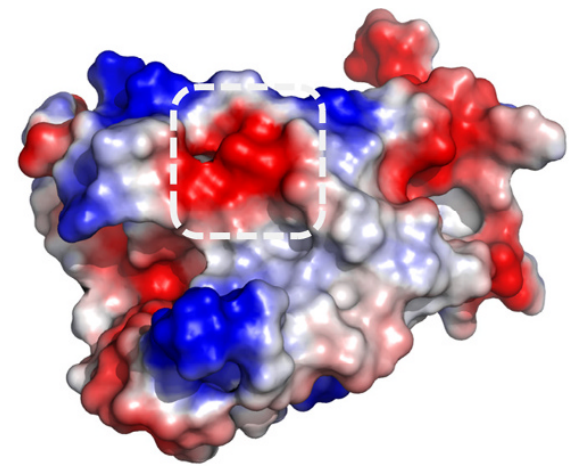

Computer model of the canine prion protein. Image adapted from PLoS Pathog. 13, e1006716 (2017).

In a paper published in PLoS Pathogens (PLoS Pathog. 13, e1006716; 2017), Castilla led a series of in vitro, in silico, and in vivo experiments that indicate that canines are resistant to prion infection from several different strains. To test the theoretical limit in vitro, the team took canine brain samples from domestic dogs and attempted to force prion propagation with a seeding technique called Protein Misfolding Cyclic Amplification (PMCA). Of six common prion strains tested (originating in mice, sheep, cows, and deer), only two caused any kind of misfolding in the canine samples, and only under modified propagation techniques.

Castilla concedes that complete natural resistance is difficult to confirm, as it's improbable to test all possible strains out there, but he is confident that the in vitro results indicate canines have a unique resistance to prion diseases. To understand why, the team turned to computers. They first determined the canine prion protein's amino acid sequence and compared it to that of the dog's close genetic relative, the cat. Unlike canines, felines are easily infected by prion diseases, and the comparison revealed two amino acid polymorphisms unique to dogs. One particular difference jumped out when the team analyzed an in silico model of the canine prion's threedimensional structure: at position 163 , part of the site where one prion can bind with another, the amino acid Asparagine is swapped with either Aspartic Acid or Glutamic Acid. Castilla thinks that those amino acids alter the charge of a superficial section of the protein in a way that prevents misfolded prions from binding, and thus infecting, healthy canine ones.

To look in vivo, the team created transgenic mice that express a prion protein with an Aspartic Acid in an equivalent position to that in canines. They inoculated the transgenic and control mice with three different pathogenic murine prions, and waited. "When we went to the animals, at that time we had a lot of information in vitro and in silico," Castilla says, "We were very confident that we will have at least a very strong resistance. What we did not expect was to have complete resistance." $100 \%$ of control mice died within the expected incubation time for the given strain, but no transgenic mouse did, nor did they show any clinical signs of disease. When they were ultimately sacrificed over a year and a half later, their brains appeared healthy.

A follow-up was recently published in Molecular Neurobiology (Mol. Neurobiol.; DOI: 10.1007/s12035-017-0832-8; 2017). This time, the lab created transgenic mice that only co-expressed the substitution, meaning some susceptible wild-type murine prion protein was still present in the brain. Although eventually all of the animals did die and had similar signs of brain damage comparable to that observed in controls, the transgenic mice survived about twice as long. These results suggest the canine polymorphism can help slow the progression of prion diseases, an encouraging observation as Castilla considers the future potential of gene therapies.

To complete the canine circle, Castilla says they want to look in the opposite direction: does removing the protective properties of those particular amino acids cause canines to become susceptible? He hopes to have an answer soon.

Ellen P. Neff 